Research Article

\title{
Shear Strength Determination in RC Beams Using ANN Trained with Tabu Search Training Algorithm
}

\author{
Alireza Shahbazian (D), ${ }^{1}$ Hamidreza Rabiefar ${ }^{(D)}{ }^{2}$ and Babak Aminnejad ${ }^{3}$ \\ ${ }^{1}$ Department of Civil Engineering, Kish International Branch, Islamic Azad University, Kish Island, Iran \\ ${ }^{2}$ Department of Civil Engineering, South Tehran Branch, Islamic Azad University, Tehran, Iran \\ ${ }^{3}$ Department of Civil Engineering, Roudehen Branch, Islamic Azad University, Roudehen, Iran \\ Correspondence should be addressed to Hamidreza Rabiefar; h_rabieifar@azad.ac.ir
}

Received 11 May 2021; Accepted 18 October 2021; Published 24 November 2021

Academic Editor: Tayfun Dede

Copyright (C) 2021 Alireza Shahbazian et al. This is an open access article distributed under the Creative Commons Attribution License, which permits unrestricted use, distribution, and reproduction in any medium, provided the original work is properly cited.

\begin{abstract}
The shear failure of reinforced concrete (RC) beams is a critical issue and has attracted the attention of researchers. The specific challenges of shear failure are the numerous factors affecting shear strength, the nonlinear behavior, and the nonlinear relationship between affecting parameters and the concrete properties. This study tackles this challenge by employing Artificial Neural Network (ANN) models. Since, according to No Free Lunch theorem, the performance of optimization algorithms is problemdependent, this paper aims to assess the feasibility of modeling the shear strength of RC beams using ANNs trained with the Tabu Search Training (TST) algorithm. To this end, 248 experimental results were collected from the literature, and a feed-forward ANN model was employed to predict the shear strength. To assess its feasibility, the ANNs were also modeled using the Particle Swarm Optimization, and Imperialist Competitive Algorithms. As a traditional technique, the multiple regression model was also employed. The shear design equations of ACI-318-2019 were also investigated and compared with Tabu Search Trained ANN model. The analysis of results suggests the superiority of Tabu Search Trained ANNs in comparison to other suggested models in literature and the ACI-318-2019 design code.
\end{abstract}

\section{Introduction}

The shear strength of reinforced concrete (RC) beams can be predicted by multiple methods. Their precision, however, remains very limited because of the number and complex nature of affecting parameters such as aggregate interlock and concrete in compression region. In addition, the cross section and the axial load of the beam (if any) could have an effect on the shear strength of the RC beams [1]. The design strength and shear behavior of members are of central concern in structural design. In concrete members, there are many types of failure, and shear failure is one of the most critical and unwanted types of failure because of the fragility of concrete systems. Thus, to withstand shear failure, RC members are employed [2]. The literature and concrete codes suggest many empirical formulas for RC beam strength. In structural design, the American Concrete
Institute (ACI) code has been used extensively. Furthermore, each of the analytical formulas proposed in concrete codes delivers good results for a given data set only [2].

There have been numerous studies on the use of soft computing techniques, especially artificial neural networks (ANNs) to assess properties of concrete. Artificial neural network models have proved to be outstanding for determination of shear strength of RC beams as shown by Mansour et al. They employed 176 experimental results and built a 9-input ANN. For the 176 test results, they achieved a 1.003 experimental-to-predicted shear strength ratio. The results indicate that ANNs have the potential to be used as a practical tool in determining the ultimate shear strength of reinforced concrete beams with stirrups in the range of parameters studies [3]. Olalusi and Awoyera investigated the shear failure in reinforced concrete beams by utilizing machine learning techniques. They employed Gaussian 
process regression and random forest techniques to develop an ML-based model high accuracy [4]. ANNs have also been shown to be accurate in predicting the shear strength of concrete beams with stirrups. Based on the observed behavior, Cladera and Marí proposed new design equations for normal and high-strength concrete beams [5]. Abdalla et al. employed the shear strength, compressive strength, shear and longitudinal reinforcement, beam depth and width, and shear span-to-depth ratio parameters to predict and model the shear strength of RC beams using the artificial neural networks. The effective parameters help guide further research in narrowing parameter list. They modeled a backpropagation-based ANN with various transfer functions and provided shear response surfaces and curves [6]. Although being fast, the backpropagation using gradient descent technique is prone to get stuck in local optima and therefore not suitable in predicting the best performing model. To predict the torsional strength of reinforced concrete beams, Arslan collected 76 test results published in the literature and based the ANN models on the compressive strength, beam cross section area, steel ratio of longitudinal bars and stirrups, closed stirrup dimensions, reinforcement yield strengths, stirrup spacing, and cross section area for one leg of closed stirrups. The researcher concluded that ANN models predict the torsional strength of concrete beams more accurately than formulas provided in codes [7].

Oreta utilized the ANN model to measure the effect of size on the shear strength of RC beams without stirrups. The researcher modeled an ANN using five input variables and concluded that the ANN model performs superior to existing equation [8]. To predict the shear strength of RC joints, Naderpour and Nagai employed seven input variables to develop an ANN model. They compared the developed model with existing equations and calculated the relative importance of input parameters on the shear strength by employing a sensitivity analysis. The results indicated that the reinforcement ratio is the most influential parameter on shear strength of RC joints [9], which is helpful in guiding further research in selecting effective parameters. Feng and Fu predicted the shear strength of internal RC beam-column joints by developing a gradient boosting regression tree built by integrating several machine learning models including ANNs and support vector machines. The researchers concluded that the utilized model predicts the shear strength more accurately than the existing models [10]. Jeon et al. proposed a multivariate adaptive regression splines model for predicting the shear strength of RC beam-column joints. They compared the model against symbolic regression and multivariate linear regression models and showed that the proposed model is more accurate [11]. Salehi and Burgueño investigated the use of Artificial Intelligence (AI) techniques in structural engineering. They evaluated traditional machine learning methods, deep learning, and pattern recognition techniques for the structural engineering use case [12]. The results of their analysis make the case for using AI techniques in structural engineering problems.

Despite their utility and attractiveness, RC deep beams are challenging to design, because various parameters nonlinearly affect their behavior and shear strength.
Remarkably, shear stress is a dominant failure mode of RC deep beams that tends to result in sudden, severe collapse and human loss [13]. To predict the shear strength of deep reinforce concrete beams, Zhang et al. employed the support vector regression method hybridized with the genetic algorithm. The model inputs were the mechanical, material, and dimensional properties of beam. In comparison to ANN and gradient boosted tree models, the employed model proved to be more accurate for shear strength prediction of deep RC beams [14]. Although being powerful, the genetic algorithm method requires a lot of trial-and-error to find the optimum values of hyperparameters and therefore is used less often. Shahnewaz et al. reviewed the proposed methods for predicting the shear strength of deep reinforced concrete beams in the literature. They concluded that the design equations are conservative in predicting the shear strength of deep RC beams. The researchers proposed an improved model based on genetic algorithm and reliability analysis [15]. This makes the case for further investigation of shear strength using contemporary techniques to get a clearer picture of the phenomenon. To predict the shear strength of prestressed and reinforced deep concrete beams, Pal and Deswal employed a support vector regression-based model. To evaluate the model accuracy relative to other models, they additionally used a backpropagation-based ANN and three empirical models from the literature. The researchers concluded that the support vector regression-based approach performance is superior to empirical and ANN models [16]. Chou et al. combined the smart artificial firefly colony algorithm with least squares support vector regression method to predict the shear strength of deep $\mathrm{RC}$ beams. The researchers calibrated the proposed model by using a dataset of experimental data collected from literature. The comparison of the model with existing equations leads researchers to conclude that the proposed method performs more accurately than the methods proposed in the literature for predicting the shear strength of deep RC beams [17]. In the realm of metaheuristic approaches, Gandomi et al. employed a hybrid search algorithm combined with genetic programming and simulated annealing, called the genetic simulated annealing for predicting the shear strength of deep $\mathrm{RC}$ beams. The comparison of the proposed model with ACI and CSA codes indicated the superiority of the method relative to these codes [18]. For shear strength of deep RC beams, Sanad and Saka proposed a predictive model based on artificial neural networks. The researchers concluded that ANN model performs more accurately than empirical equations [19]. Prayogo et al. utilized a hybrid model composed of two support vector machine models and symbiotic organisms search algorithm, called optimized support vector machines with adaptive ensemble weighting to predict the shear strength of deep RC beams. The researchers demonstrated the feasibility of the proposed algorithm in predicting the shear strength of deep reinforced concrete beams [13].

The purpose of this study is to assess the feasibility of using Tabu Search Training (TST) algorithm to calibrate the weights of an artificial neural network model for predicting the shear strength of reinforced concrete beams. The input 
parameters of the model include physical, geometric, and material properties. The dataset utilized is composed of 248 experimental results collected from literature. Section 2 summarizes the artificial neural network model and the tabu search training algorithm in a general context. The model training and development are detailed in Section 3, followed by results and conclusion in Sections 4 and 5 . The general outline of the paper is given in Figure 1 [20].

\section{Background}

2.1. Artificial Neural Networks. As one of the most lively fields of current research, Artificial Neural Networks (ANNs) offer valuable features and capabilities including learning and adapting to existing knowledge, generalization, parallel processing, and therefore higher processing speed, and high error tolerance [21-23]. The bioinspired feedforward artificial neural network is an algorithm that consists of neurons organized in layers. Each neuron in a layer is connected to all neurons of the previous layers. The signals are transmitted between neurons through connection lines, and the weight of each connection shows its strength. In fact, the knowledge of the ANN is stored using these weights. To evaluate the output of each neuron, an activation function is applied to the sum of its weighted inputs (plus a bias value). The information flows move from the input neurons and through the hidden layers to the output neurons layer by layer [24].

The weights of an ANN are typically randomly initialized, and the network output is thus different from the target values. The weights and biases of the network need to be optimized in a process called neural network training to minimize the model error $[25,26]$. Generally, the strategies for addressing this problem of optimization can be divided into two categories: gradient-based and metaheuristic. Gradient-based methods are quick; however, they can get stagnated in local minima. The algorithm does not get stuck in the local minima using metaheuristic methods; however, the solution obtained is not necessarily the global minimum. The metaheuristic approaches are designed to explore and exploit the solution space to provide accurate results [25-27]. Figure 2 displays an ANN with 9 neurons in the input layer, one hidden layer with 16 neurons, and one output layer. This is the top performing architecture utilized in this study.

2.2. Tabu Search Training Algorithm. Tabu search (TS) is a discrete optimization algorithm proposed by Glover in 1986 [28], and its modern form is ascertained by the researcher in 1989 and 1990 [29, 30]. Being a metaheuristic optimization algorithm, it is designed to guide the search operator from being trapped in local minima. It employs a short-term memory to move in the search space by a controlled randomization and thus avoid cycling on previously visited solutions by setting as tabu every step that has led to an improvement of the cost function and a long-term memory to diversify and explore the search space [31, 32].
Additionally, intermediate-term memory structures could be introduced to lead moves toward promising areas $[28,33]$.

To make it usable for training artificial neural networks, Dengiz et al. [31] introduced a modified version of Tabu Search algorithm later on. The proposed algorithm is named Tabu Search Training (TST) algorithm. TST commences by generating an initial vector of weights, i.e., $W_{\text {cur }}$ for the artificial neural network. It then generates a neighborhood around the current solution by changing one of the elements $\left(w_{i}\right)$ of $W_{\text {cur }}$ by a randomly generated number drawn from a uniform distribution. The weight $w_{i}$ is then changed by the amount $v_{i, j}$ if the value of $v_{i, j}$ is not in a tabu-value-start list for $V$ (tabu value list size) iterations. Each weight $w_{i}$ is updated $K$ times resulting in $n K$ number of neighbor weight vectors, where $n$ is the size of weights vector $W_{\text {cur. }}$. Then, for each of the neighbors, the cost function is evaluated, and if the move is allowed through the tabu mechanism, the weights vector $W_{\text {cur }}$ is updated, and if the move is not allowed, the next best neighbor in terms of cost function is chosen if allowed.

The tabu search training algorithm employs a short-term memory to allow for intensification of search and a longterm memory to allow for diversification of search and thus avoiding local minima. The details of the algorithm are given in Dengiz et al. [31]. The outline of the TST algorithm is depicted in Figure 3.

\section{Methods and Materials}

3.1. Dataset. The database in this paper is based on data collected by Baghi and Barros [1]. The collected data consist of 248 experimental samples gathered from numerous published papers [34-44]. Baghi and Barros identified 9 effective parameters on the shear strength of reinforced concrete T-beams, namely, flange width $(b)$, flange thickness $\left(h_{f}\right)$, concrete compressive strength $\left(f_{c}^{\prime}\right)$, web width $\left(b_{w}\right)$, effective depth $(d)$, yield stress of stirrups times transverse reinforcement ratio $\left(f_{\mathrm{st}, y} . \rho_{\mathrm{st}}\right)$, shear span-to-depth ratio $(a /$ $d)$, flexural reinforcement ratio $\left(\rho_{\mathrm{sl}}\right)$, and $k_{f}$ coefficient that takes the influence of flanges of the T-beam on shear capacity into account and is defined by using Equations (1) and (2) $[1,45]$.

$$
k_{f}=1+n \cdot\left(\frac{h_{f}}{b_{w}}\right) \cdot\left(\frac{h_{f}}{d}\right) \leq 1.5,
$$

where

$$
n=\frac{b-b_{w}}{h_{f}} \leq 3 .
$$

The descriptive statistics of experimental dataset are given in Table 1 . The histogram of the shear strength of reinforced concrete T-beams is displayed in Figure 4.

Various ranges of input variables to an artificial neural network (ANN) may have unfavorable effects on the model such as optimization algorithm divergence and an added training time [25]. Therefore, the input and output variables 


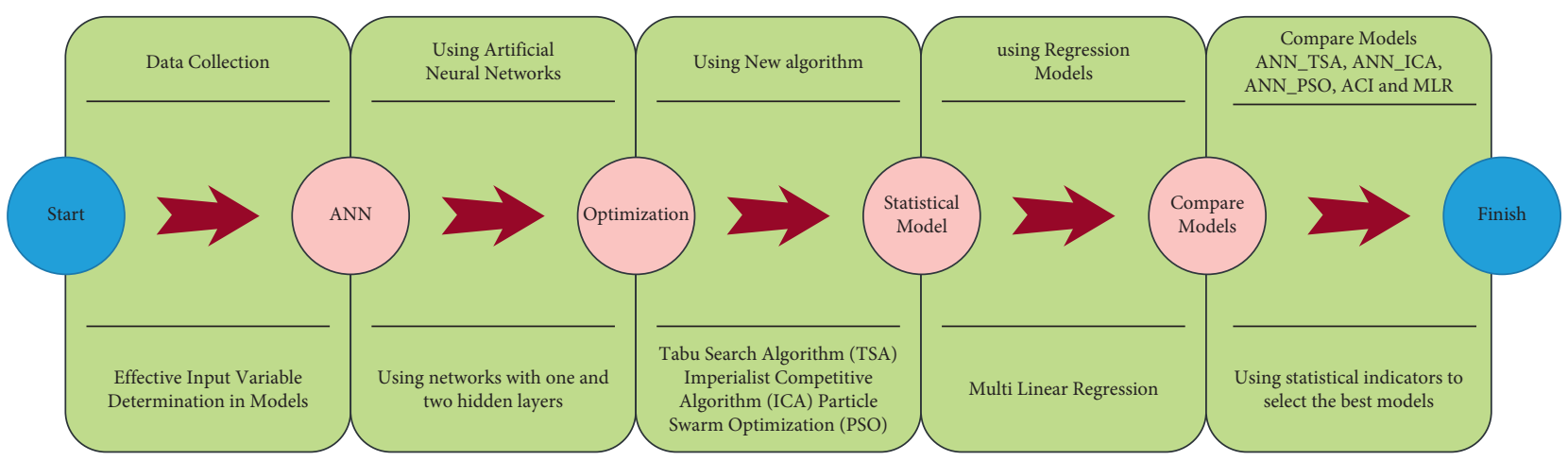

FIgUre 1: Outline of the paper.

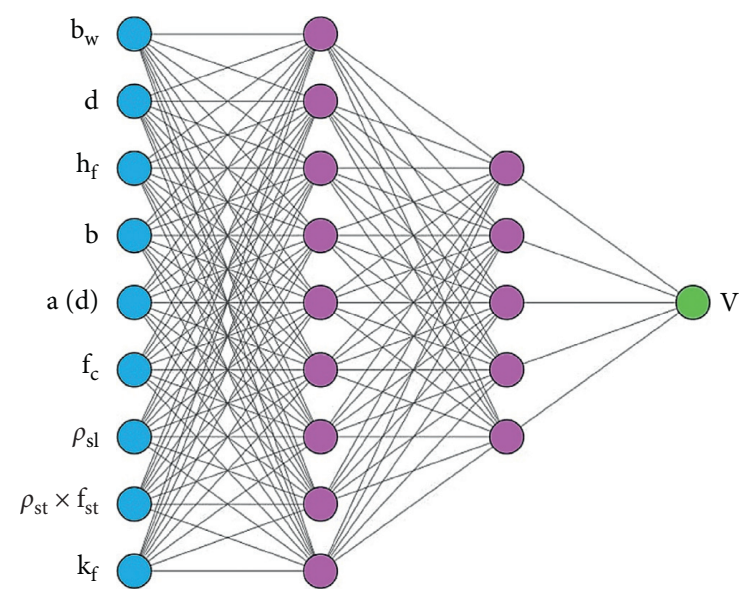

FIgURE 2: Architecture of the optimum artificial neural network used in this study with 9 input neurons, 16 neurons in the hidden layer, and one neuron in output layer.

of the dataset were transformed (normalized) into a range of [-1 1] using Equation (3) as follows:

$$
X_{n}=\frac{2\left(X-X_{\min }\right)}{X_{\max }-X_{\min }}-1,
$$

where $X_{n}$ is the normalized value of the variable, $X_{\max }$ is the maximum, and $X_{\min }$ is its minimum value. $X$ is the original (nontransformed) value of the variable. The minimum and maximum values of each of the 9 input parameters and the target value of shear strength are given in Table 1. Since ANN is trained on normalized data, it should be noted that when using ANN, the network should be fed with normalized values of variables, and the output of the network should be denormalized, i.e., transferred into its original range [46].

3.2. Performance Measures. The benchmarks by which the model's performance and prediction accuracy are evaluated should be described in order to evaluate and compare models. The performance measure selected is the fitness value (or cost) of objective function on test data. The explanation for the performance evaluation based on test data is the selection of a model with the most generalization capability.
The statistical measures used to evaluate the performance of different predictive models are the Mean Error (ME), Mean Absolute Error (MAE), Mean Squared Error (MSE), Root Mean Squared Error (RMSE), Average Absolute Error (AAE), Model Efficiency (EF), and Variance Account Factor (VAF) that are defined as Equations (4)-(8) [47]:

$$
\begin{aligned}
\mathrm{ME} & =\frac{1}{n} \sum_{i=1}^{n}\left(P_{i}-O_{i}\right), \\
\mathrm{MAE} & =\frac{1}{n} \sum_{i=1}^{n}\left|P_{i}-O_{i}\right|, \\
\mathrm{MSE} & =\frac{1}{n} \sum_{i=1}^{n}\left(P_{i}-O_{i}\right)^{2}, \\
\mathrm{RMSE} & =\left[\frac{1}{n} \sum_{i=1}^{n}\left(P_{i}-O_{i}\right)^{2}\right]^{1 / 2}, \\
\mathrm{AAE} & =\frac{\left|\sum_{i=1}^{n}\left(O_{i}-P\right) / O_{i}\right|}{n} \times 100 .
\end{aligned}
$$

3.3. Empirical Model Development Using ANNs and TSO. As mentioned in Section 3.1, there are 9 parameters influencing the shear strength of reinforced concrete T-beams. So, the artificial neural networks trained will all have 9 neurons in the input layer, and one neuron in the output layer as shown in Figure 2. Feed-forward networks, as described in Section 2.1, were used for ANNs.

The overfitting phenomenon affects artificial neural networks. An overfitted network is highly accurate in the training phase but is unable to produce a good performance on test data not encountered before and therefore does not have generalization capability. In order to minimize the overfitting effects, data were randomly divided into two sets, as recommended in literatures $[26,46] .70 \%$ (174 cases) were used to train the network, and the remaining 30\% (74 cases) were used to test the performance of the network.

The number of hidden layers and total neurons in hidden layers is problem-dependent for an artificial neural network 


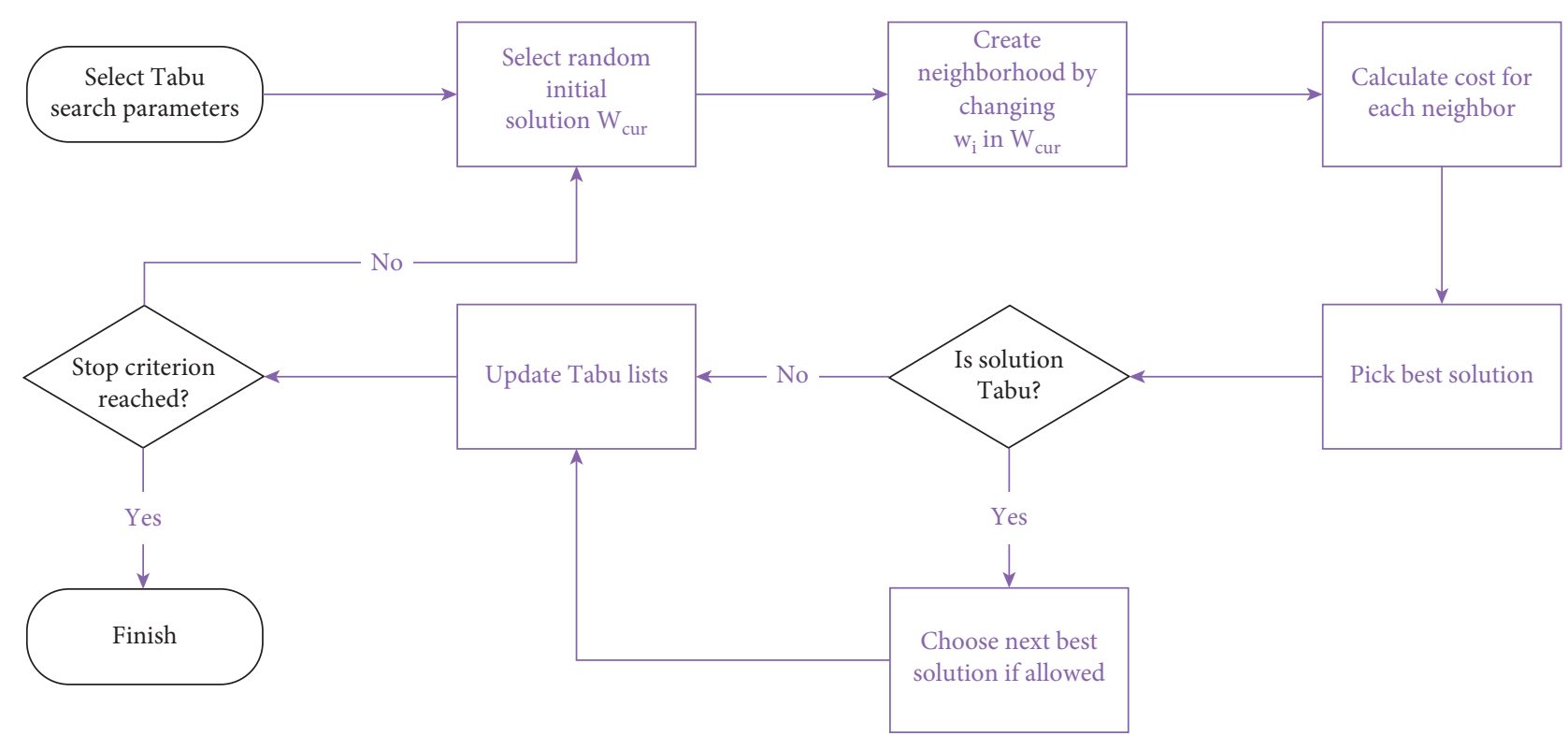

FIGURE 3: Flowchart of the tabu search training algorithm.

Table 1: Descriptive statistics of collected experimental data.

\begin{tabular}{|c|c|c|c|c|c|c|}
\hline Parameter & Unit & Type & Max & Min & Average & STD \\
\hline$b_{w}$ & $\mathrm{~mm}$ & Input & 457.00 & 50.00 & 204.21 & 89.20 \\
\hline$d$ & $\mathrm{~mm}$ & Input & 1200.00 & 198.00 & 425.21 & 217.44 \\
\hline$h_{f}$ & $\mathrm{~mm}$ & Input & 152.00 & 0.00 & 36.95 & 47.37 \\
\hline$b$ & $\mathrm{~mm}$ & Input & 1200.00 & 125.00 & 387.14 & 222.27 \\
\hline a/d & & Input & 5.40 & 2.53 & 3.45 & 0.56 \\
\hline$f_{\mathrm{c} 0}$ & $\mathrm{MPa}$ & Input & 125.00 & 10.00 & 44.01 & 22.47 \\
\hline$\rho_{\mathrm{sl}}$ & $\%$ & Input & 15.61 & 0.49 & 3.10 & 2.16 \\
\hline$\rho_{\mathrm{st}} \times f_{\mathrm{st}, y}$ & - & Input & 9.60 & 0.00 & 1.18 & 1.48 \\
\hline$k_{f}$ & - & Input & 1.50 & 1.00 & 1.10 & 0.13 \\
\hline$V$ & $\mathrm{kN}$ & Output & 1330.00 & 33.00 & 255.93 & 224.12 \\
\hline
\end{tabular}

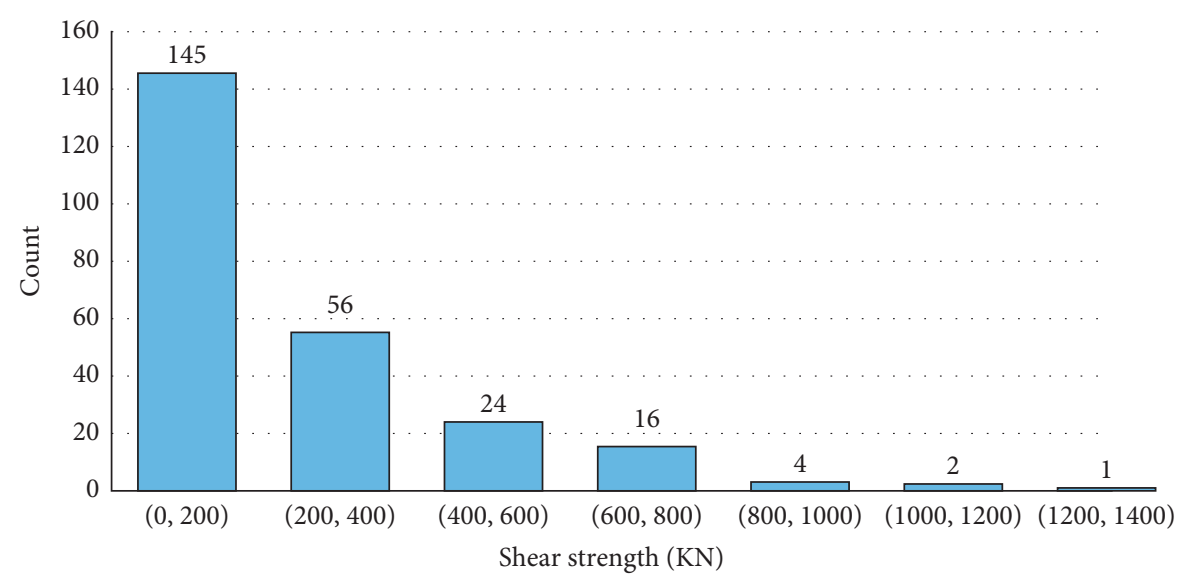

FIgURE 4: Shear strength distribution of experimental data.

model [48]. A trial-and-error approach was therefore used to obtain the optimal architecture, i.e., the architecture that best represents data. Equation (9) provides a commonly used heuristic for the total number of neurons of an artificial neural network by using Equation (9) [49]:

$$
N_{H} \leq 2 N_{I}+1
$$

where $N_{H}$ is the number of hidden layer nodes, and $N_{I}$ is the number of inputs. Since the number of effective parameters is 9 , the empirical equation suggests that the number of 
hidden layer nodes can be chosen to be less than 19 . Therefore, different architectures having one and two hidden layers and less than a total of 19 neurons were trained. A total of 30 different ANN architectures were trained, and the selected architectures are given in Table 2. The hyperbolic tangent and the logistic functions were chosen as the activation function of the hidden layers, and identity function was chosen as the activation function of the output layer.

The process of adjusting the weights and biases of an artificial neural network, i.e., training, is a minimization process, where the optimal solution is one with weights and biases that minimize the cost function, i.e., the prediction error of the network. To this end, the tabu search training (TST) algorithm described in Section 2.2 was applied. MATLAB [50] software package was used to code the ANNs and tabu search training algorithm. The optimum parameters of the tabu search training algorithm were found using trial-and-error, and the parameters used for training various ANN architectures are given in Table 3.

\section{Results}

4.1. Empirical Model Evaluation. As described in Section 3, 30 different architectures of artificial neural networks (ANNs) having one and two hidden layers were trained using the tabu search training (TST) algorithm. The networks had the hyperbolic tangent, and logistic function as the activation function of the hidden layers, and the activation function of the output layer was chosen to be the identity function. In the remaining sections, the ANNs will be referred to by designation ANN-ALG $n L\left(n_{1}-n_{2}\right)$, where ALG designates the training algorithm used, $n L$ signifies that the ANN has $n$ hidden layers, and $n_{1}$ and $n_{2}$ designate the number of neurons in the first and second hidden layers, respectively.

Of the 30 models trained to predict the shear strength of reinforced concrete T-beams, the top four based on their values of Mean Squared Error (MSE) were chosen. The ANNs along with their performance measures on training data are given in Table 4, and the performance measures on testing data of the same top four networks are given in Table 5.

Referring to Table 5, the network ANN-TST 2L (9-5) has the least values of MSE (and RMSE) for testing data; therefore, it is chosen as the top model trained using the TST for further analysis. The activation function of this model is the hyperbolic tangent function. On training data, ANNTST 2L (9-5) has AAE, MSE, $R^{2}$, RMSE, and MAE values of $0.07,520.70,0.9903,22.82$, and 14.16 , respectively.

For testing data, ANN-TST 2L (9-5) has AAE, MSE, $R^{2}$, RMSE, and MAE values of 0.16, 2217.08, 0.9475, 47.09, and 32.72 , respectively. It should be noted that the error metrics for training and testing data were calculated using the data values in the original range of the variables and not in the normalized range of $[-11]$ as is sometimes used in literature.

For a visual representation of the performance of ANNTST 2L (9-5), the predicted values of the empirical model vs. their values found from experiment are depicted in Figures 5-7 for training data, testing data, and all data,
TABLE 2: Trained artificial neural network architectures.

\begin{tabular}{lccccc}
\hline Num & Topology & Num & Topology & Num & Topology \\
\hline 1 & $4-4$ & 11 & $6-4$ & 21 & $8-4$ \\
2 & $4-5$ & 12 & $6-5$ & 22 & $8-5$ \\
3 & $4-6$ & 13 & $6-6$ & 23 & $8-6$ \\
4 & $4-7$ & 14 & $6-7$ & 24 & $8-7$ \\
5 & $4-8$ & 15 & $6-8$ & 25 & $8-8$ \\
6 & $5-4$ & 16 & $7-4$ & 26 & $9-4$ \\
7 & $5-5$ & 17 & $7-5$ & 27 & $9-5$ \\
8 & $5-6$ & 18 & $7-6$ & 28 & $9-6$ \\
9 & $5-7$ & 19 & $7-7$ & 29 & $9-7$ \\
10 & $5-8$ & 20 & $7-8$ & 30 & $9-8$ \\
\hline
\end{tabular}

Table 3: Optimum tabu search algorithm parameters used in training artificial neural network models.

\begin{tabular}{lcc}
\hline Algorithm & Parameter & Value \\
\hline & Maximum iterations & 100 \\
& $K$ & 2 \\
Tabu search algorithm & Lower bound & 0 \\
& Upper bound & 0.001 \\
& Tabu value list size & 10 \\
& Bound increment & 0.001 \\
& Tabu increase decrease list size & 12 \\
& Tabu frequency list size & 5 \\
& Maximum frequency & 10 \\
\hline
\end{tabular}

TABLE 4: Error metrics of the top four artificial neural networks on training data.

\begin{tabular}{lcccc}
\hline Network designation & MAE & MSE & RMSE & AAE \\
\hline ANN-TST 2L (9-5) & 14.16 & 520.70 & 22.82 & 0.07 \\
ANN-TST 2L (6-8) & 16.73 & 590.98 & 24.31 & 0.09 \\
ANN-TST 2L (9-4) & 62.82 & 8292.82 & 91.06 & 0.34 \\
ANN-TST 2L (5-7) & 22.90 & 1117.06 & 33.42 & 0.11 \\
\hline
\end{tabular}

TABle 5: Error metrics of top four artificial neural networks on testing data.

\begin{tabular}{lcccc}
\hline Network designation & MAE & MSE & RMSE & AAE \\
\hline ANN-TST 2L (9-5) & 32.72 & 2217.08 & 47.09 & 0.16 \\
ANN-TST 2L (6-8) & 38.40 & 4281.70 & 65.43 & 0.16 \\
ANN-TST 2L (9-4) & 67.37 & 7757.97 & 88.08 & 0.37 \\
ANN-TST 2L (5-7) & 45.63 & 8438.66 & 91.86 & 0.19 \\
\hline
\end{tabular}

respectively. It is evident that the values predicted by the model are close to the line $y=x$, signifying the accuracy of the model. The comparison between experimental results and ANN-TST 2L (9-5) predictions on testing data is displayed in Figure 8.

4.2. Comparison with Other Methods. To evaluate the performance of tabu search training algorithm for training an artificial neural network for the purpose of predicting the shear strength of reinforced concrete T-beams, three different models are also developed. One artificial neural 


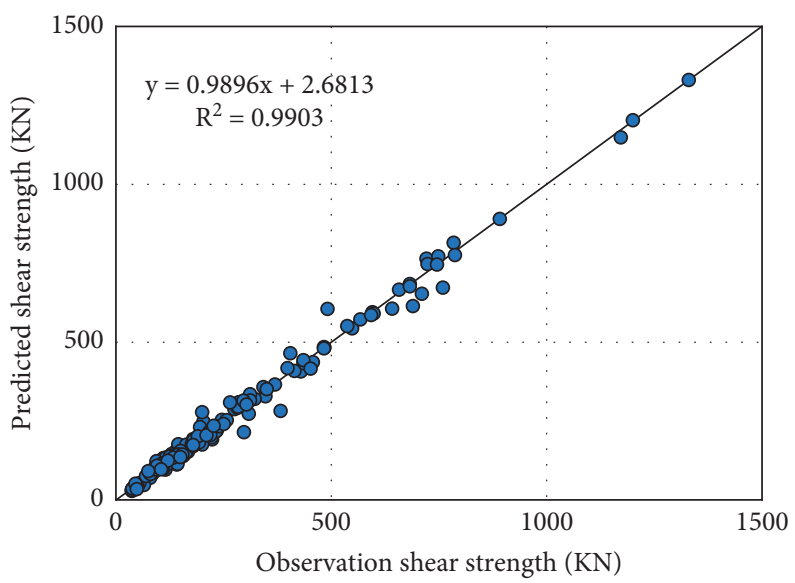

- ANN-TST 2L (9-5)

FIgURE 5: Target (experimental) versus predicted values of shear strength for ANN-TST 2L (9-5) model using training data.

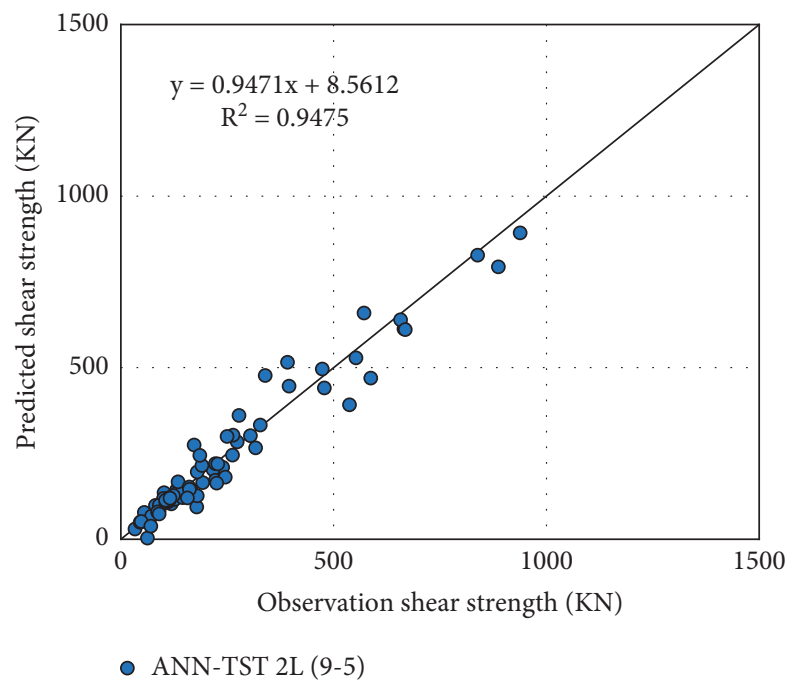

FIgURE 6: Target (experimental) versus predicted values of shear strength for ANN-TST 2L (9-5) model using testing data.

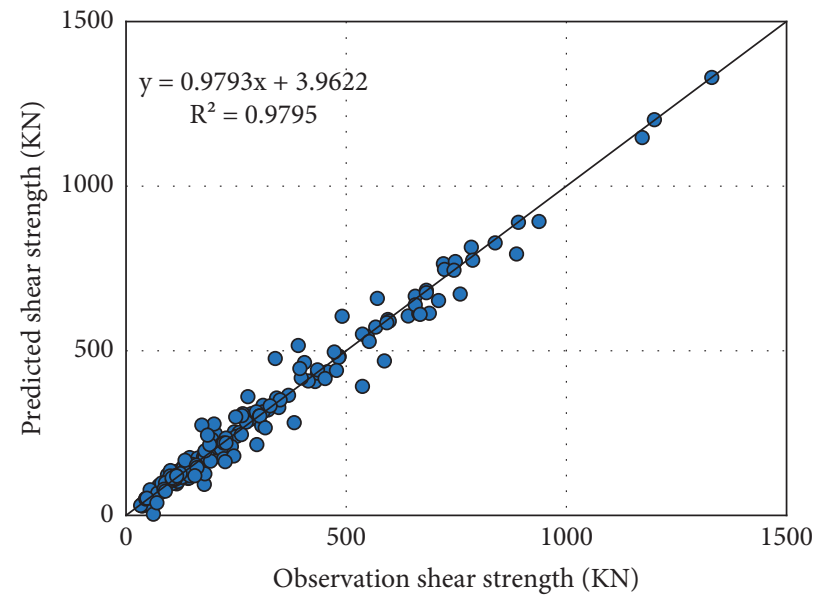

- ANN-TST 2L (9-5)

FIgURE 7: Target (experimental) versus predicted values of shear strength for ANN-TST 2L (9-5) model using all data. 


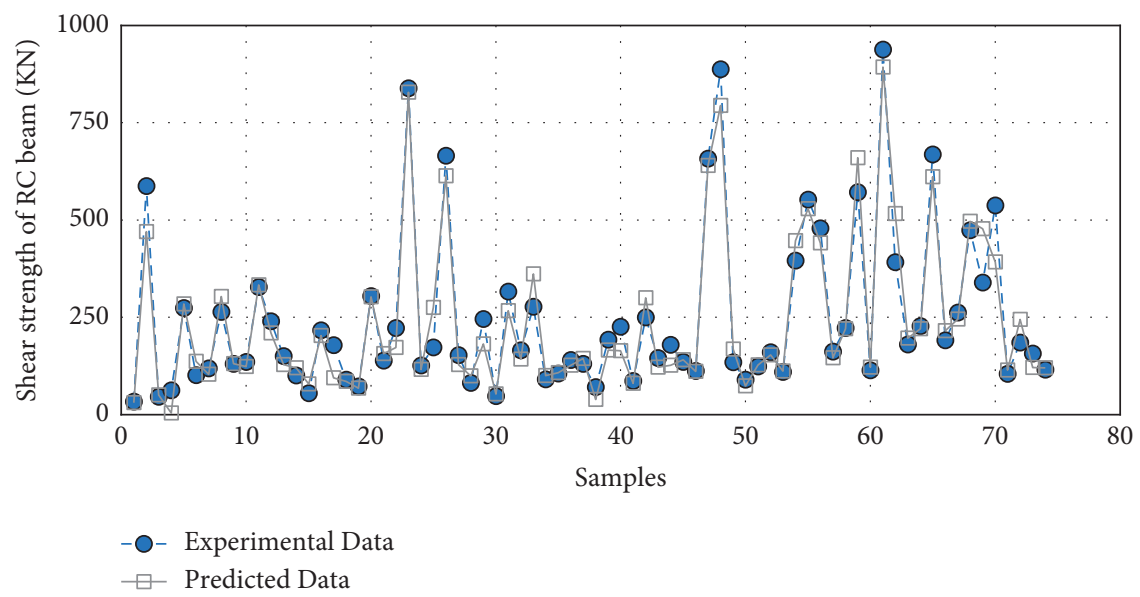

Figure 8: Comparison between the experimental results and ANN-TST 2L (9-5) predictions for testing data.

network model is trained using the imperialist competitive algorithm (ICA), one trained using the particle swarm optimization (PSO) algorithm, one multivariable linear regression model, and ACI standard code.

4.2.1. Imperialist Competitive Algorithm Model. The ANNICA 2L (9-5) architecture was trained using the imperialist competitive algorithm (ICA), and the optimum ICA parameters used to train the network were found by trial-anderror, and their values are given in Table 6. Compared to TST-trained neural network, this network has much higher prediction error. For a visual representation of the performance of this model, the predicted values of shear strength vs. their experimental values are shown in Figure 9. It is evident that the points on the plot are much farther from the $y=x$ line in comparison to the ANN-TST 2L (9-5) model.

4.2.2. Particle Swarm Optimization Model. The same ANNPSO 2L (9-5) architecture was used to train the network using particle swarm optimization (PSO) algorithm, the optimum PSO parameters used to train the network were found by trial-and-error, and their values are given in $\mathrm{Ta}$ ble 6. Compared to ICA-trained network, this model performs relatively better; however, compared to TST-trained neural network, it has much higher prediction error. The performance of this model is visually represented in Figure 10 that depicts the predicted values of shear strength vs. their experimental values.

4.2.3. Multiple Regression Model. A Multiple Linear Regression (MLR) model [51] was developed as a classical model to provide an easy-to-use model. The model was developed in Minitab 19 software package using the same data. The influence of each variable can be approximated by checking the values of regression coefficients [52, 53]. The resulting regression equation (10) is

$$
V=560+\left(1.17 b_{w}\right)+(0.144 d)+\left(2.27 h_{f}\right)+(0.0296 b)-(26.9 a / d)+\left(1.35 f_{c}^{\prime}\right)+\left(25.9 \rho_{\mathrm{sl}}\right)+\left(78.5 \rho_{\mathrm{st}} \cdot f_{\mathrm{st}, y}\right)-763 k_{f} .
$$

where $\mathrm{b}$ is the flange width, $h_{f}$ is the flange thickness, $f_{c}^{\prime}$ is the concrete compressive strength, $b_{w}$ is the web width, $d$ is the effective depth, $f_{\text {st }, y} . \rho_{\text {st }}$ is the yield stress of stirrups times transverse reinforcement ratio, a/d is the shear span-todepth ratio, $\rho_{\mathrm{sl}}$ is the flexural reinforcement ratio, and $k_{f}$ is the coefficient.

4.2.4. ACI-318. The model presented by the ACI 318-14 standard code estimates the shear strength of RC beams. However, it does not provide any information concerning their rotation capability. In this case, the nominal shear resistance is reached:

$$
\left(V_{c}=\varnothing \times 0.17 \times \gamma \times f_{c}^{\prime} \times 0.5 \times b_{w} \times d\right),
$$

where $V_{c}$ is the shear resistance; $\varnothing=0.75 ; \lambda=0.75$ for lightweight concrete and 1 for normal weight concrete; $f_{c}$ is the 28- $\mathrm{d}$ cylinder compressive strength of concrete in $\mathrm{MPa}$; $b_{w}$ is the web width; $d$ is the effective depth of the beam in $\mathrm{mm}$.

Compared to TST-trained, PSO-trained, and ICAtrained ANNs, multiple linear regression model performs far less accurately. The experimental vs. predicted values of these models on all data are depicted in Figures 10-12.

A useful diagram for evaluating the performance of ANN-TST is the Taylor diagram depicted in Figure 13. The Taylor diagram depicts the adequacy of model based on root mean square centered difference, the correlation coefficient, and the standard deviation [54]. 
TABLE 6: Optimum ICA and PSO parameters used in training artificial neural network models.

\begin{tabular}{lcccc}
\hline \multicolumn{2}{c}{ Optimum parameters of imperialist competitive algorithm } & \multicolumn{3}{c}{ Optimum parameters of particle swarm } \\
optimization
\end{tabular}

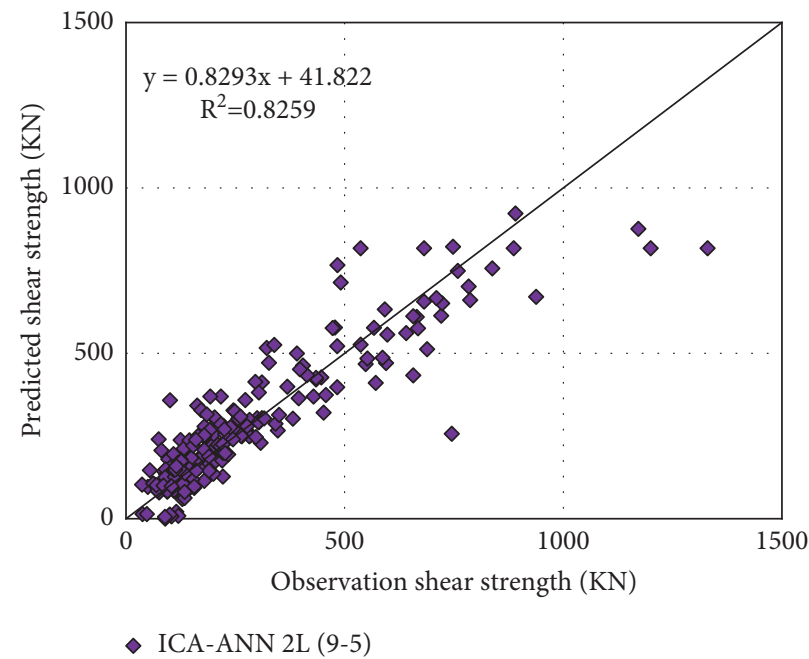

Figure 9: Target (experimental) versus predicted values of shear strength for ANN-ICA 2L (9-5) model using all data.

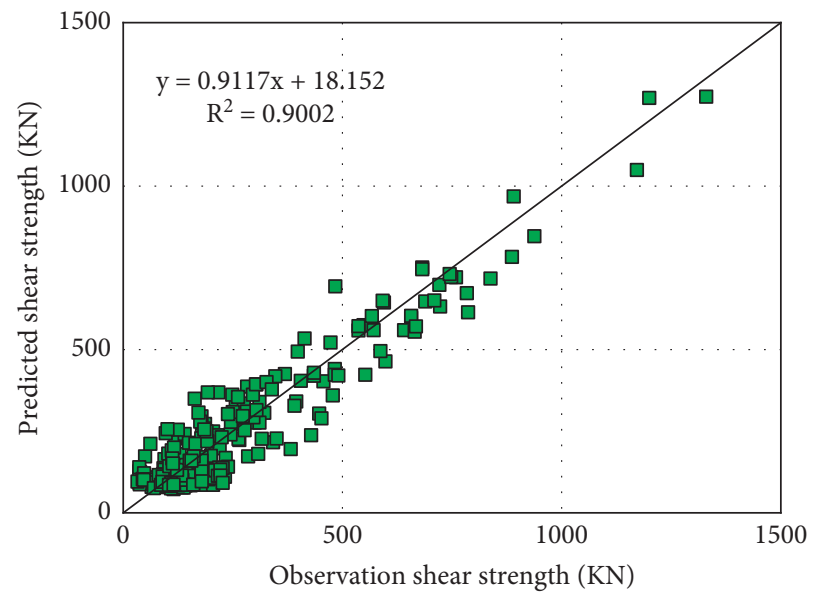

口 PSO-ANN 2L (9-5)

FIGURE 10: Target (experimental) versus predicted values of shear strength for ANN-PSO 2L (9-5) model using all data.

The statistical indices of ME, MAE, MSE, RMSE, AAE, $\mathrm{EF}$, and VAF on all data for ANN-TST 2L (9-5), ANN-PSO 2L (9-5), ANN-ICA 2L (9-5), ACI, and the multiple linear regression model are given in Table 7 . Comparing these values, the top performing empirical model is the ANN-TST $2 \mathrm{~L}$ (9-5), the second-best performing model is the ANN-PSO 2L (9-5) model, multiple linear regression, and ANN-ICA $2 \mathrm{~L}(9-5)$, and the least accurate model is the ACI standard code.
4.3. Sensitivity Analysis. Because of the improved results in ANN-TST 2L (9-5) model relative to ANN-PSO 2L (9-5), ANN-ICA 2L (9-5), and multiple linear regression model, the sensitivity analysis for determining the relative contribution of each of the 9 input variables was performed using this analytical model. The profile method proposed by Lek [53, 55] was implemented in MATLAB [50] software package and used for sensitivity analysis. This approach is based on analyzing each of the inputs while holding others 


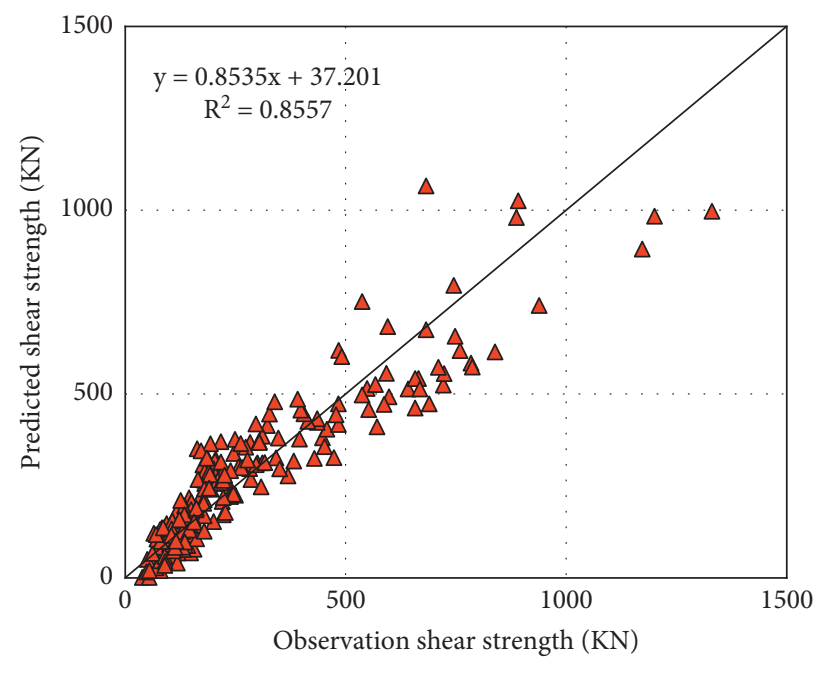

$\triangle$ MLR

FIgURE 11: Target (experimental) versus predicted values of shear strength for multiple linear regression model using all data.

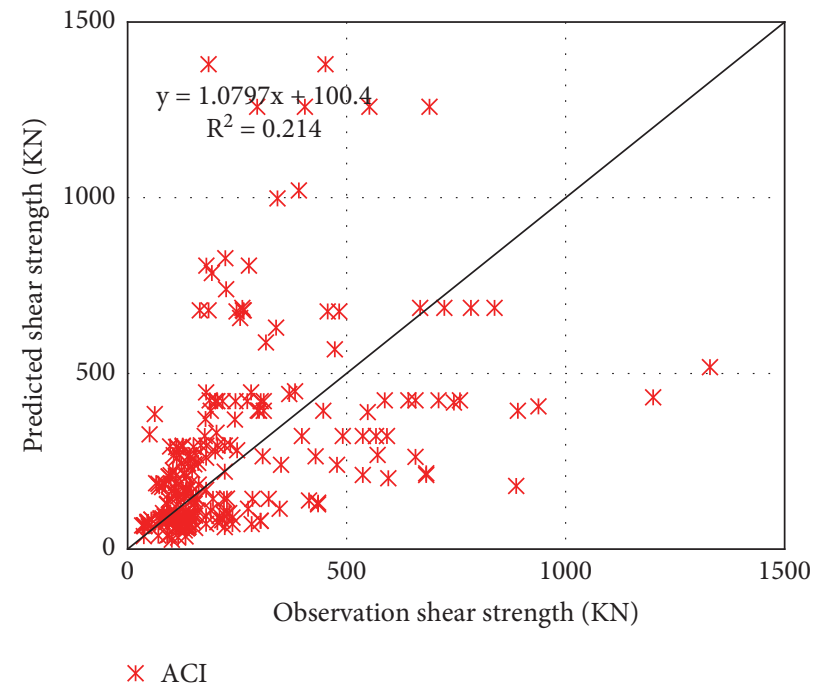

Figure 12: Target (experimental) versus predicted values of shear strength for ACI-318 model using all data.

fixed. The input variable ranges were divided into several equal intervals called the scale. While setting the values of other variables to $m$ different fixed values, the output of the network was calculated for the entire range of the selected variable, which resulted in $m$ groups of outputs. Eventually, $m$ groups of outputs were combined by calculating the median output for every single input case. The fixed values selected for each of the variables were their min, Q1, median, Q3, and max. The implementation and the theory are clearly explained by Lek [53, 55]. In this study, the 192 scale was used as suggested by Lek $[53,55]$.

The relative importance and contribution of explanatory variables (9 inputs) on the response variable (shear strength of reinforced concrete T-beams) were calculated, and the resulting contribution is plotted in Figure 14. The most influential parameter is $\rho_{\text {st }} \times f_{\text {st }, y}$, where $25 \%$ of the variation in the response variable is attributed to it. The next three most influential parameters are $h_{f}, b_{w}$ and $d$ that are $23 \%, 22 \%$, and $15 \%$. The least influential parameters are shear span-to-depth ratio $(a / d)$ that is $1 \%$.

4.4. Predictive Model and ANN Weights. The top empirical model produced in this study is the ANN-TST 2L (9-5). This model is not useful for the end user unless its source file is provided to them. So, in this section, the weights and biases of this network are provided. As mentioned in Section 3.1, the input data to the network must be first normalized using Equation (3) and using the maximum and minimum values given in Table 1 for each variable, and the output of the network must be denormalized using Equation (14). The input is a $9 \times 1$ vector called $a^{(1)}$. The shear strength is calculated using equations (12)-(14): 


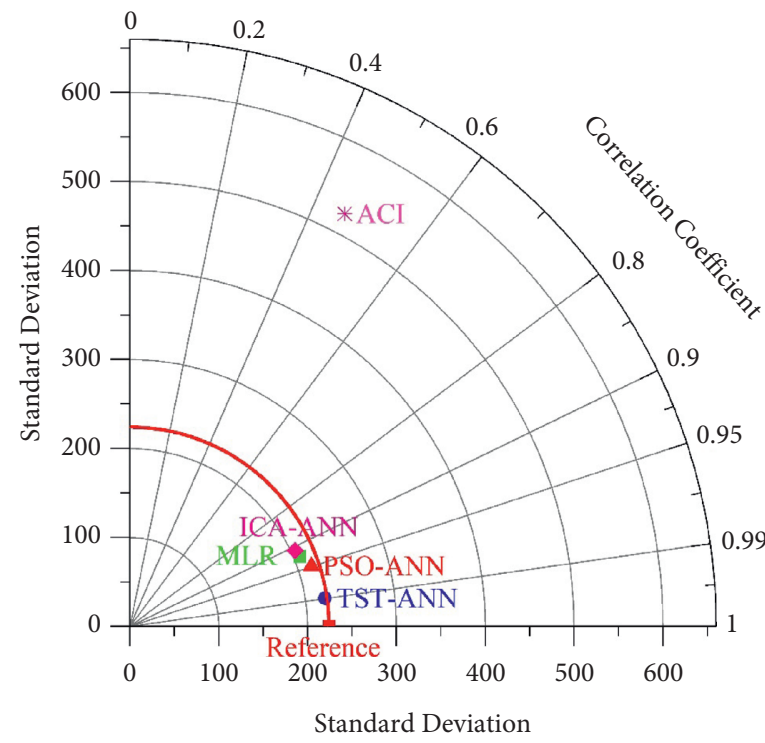

FIGURE 13: Taylor diagram visualization of model performance, in terms of shear strength.

TABLE 7: Error metrics of top ANN-TST, ANN-PSO, ANN-ICA, and MLR models on all data.

\begin{tabular}{lcccc}
\hline Network designation & RMSE & AAE & $R^{2}$ & $y=a x+b$ \\
\hline ANN-TST 2L (9-5) & 32.04 & 0.10 & 0.9795 & $y=0.9793 x+3.9622$ \\
MLR & 84.96 & 0.34 & 0.8557 & $y=0.8535 x+37.201$ \\
ACI & 478.63 & 0.96 & 0.214 & $y=1.0797 x+100.4$ \\
PSO-ANN 2L (9-5) & 70.84 & 0.34 & 0.9002 & $y=0.9117 x+18.152$ \\
ICA-ANN 2L (9-5) & 93.35 & 0.36 & 0.8259 & $y=0.8293 x+41.822$ \\
\hline
\end{tabular}

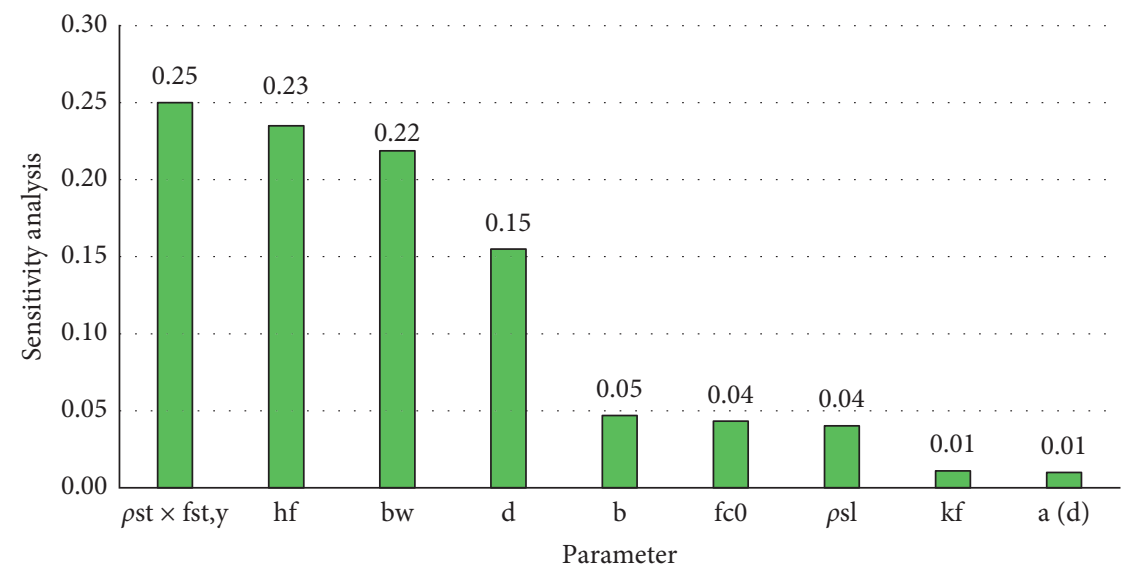

FIGURE 14: Relative contribution of input parameters on the shear strength of reinforced concrete T-beams.

$$
\begin{aligned}
a^{(2)} & =\tanh \left(\vartheta^{(1)} \times a^{(1)}+b_{1}\right), \\
v^{\text {predict (normalized) }} & =\tanh \left(\vartheta^{(2)} \times a^{(2)}+b_{2}\right), \\
v^{\text {predict }} & =\frac{v^{\text {predict }(\text { normalized })}+1}{2} \times\left(v_{\max }-v_{\min }\right)+v_{\min },
\end{aligned}
$$


where tanh is the hyperbolic tangent function, $v^{\text {predicted }}$ is the predicted value of shear strength, and $v_{\max }$ and $v_{\min }$ are the minimum and maximum shear strength values in database given in Table 1 . The weight $(\theta)$ and bias $(b)$ matrices are as follows:

$$
\begin{aligned}
& \theta^{(1)}=10^{-1} \times\left[\begin{array}{ccccccccc}
0.048 & 0.035 & -0.538 & -0.288 & -0.218 & -0.411 & 0.268 & 0.797 & -0.373 \\
-0.126 & 0.085 & 0.306 & -0.309 & 0.147 & 0.398 & -0.046 & 0.153 & -0.321 \\
0.088 & -0.269 & 0.113 & -0.462 & -0.044 & -0.188 & -0.385 & -0.653 & -0.632 \\
-0.230 & 0.360 & 0.404 & 0.281 & -0.096 & 0.076 & -0.301 & -1.320 & 0.289 \\
-0.229 & -0.151 & -0.532 & 0.062 & 0.118 & -0.559 & 0.348 & 0.721 & -0.042 \\
0.513 & 0.541 & 0.536 & 0.352 & 0.077 & 0.404 & 0.710 & 0.812 & -0.704 \\
-0.207 & -0.957 & -0.057 & -0.339 & 0.012 & 0.308 & 0.220 & 0.015 & 0.203 \\
0.184 & -0.300 & 0.291 & 0.375 & 0.254 & 0.102 & -0.030 & 0.050 & -0.268 \\
0.083 & 0.121 & 0.311 & 0.002 & 0.167 & 0.253 & -0.482 & -0.666 & -0.511
\end{array}\right] \text {, } \\
& \theta^{(2)}=10^{-1} \times\left[\begin{array}{cccccccccc}
0.459 & 0.246 & -0.737 & -0.252 & -0.543 & 0.488 & -0.239 & -0.422 & 0.100 \\
-0.005 & -0.187 & 0.354 & 0.578 & 0.415 & -0.071 & 0.459 & -0.043 & 0.093 \\
0.484 & 0.389 & -0.136 & -0.795 & -0.621 & 0.449 & -0.222 & -0.067 & 0.451 \\
-0.612 & -0.335 & 0.444 & -0.061 & -0.243 & -1.124 & -0.329 & 0.199 & 0.283 \\
-0.364 & 0.139 & 0.450 & -0.234 & -0.373 & -0.211 & 0.273 & 0.057 & -0.348
\end{array}\right] \text {, } \\
& \theta^{(3)}=10^{-1} \times\left[\begin{array}{lllll}
1.252 & -0.492 & 0.816 & -1.193 & -0.313
\end{array}\right] \text {, }
\end{aligned}
$$

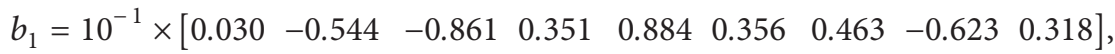

$$
\begin{aligned}
& b_{2}=10^{-1} \times\left[\begin{array}{lllll}
0.935 & 0.172 & 0.535 & -0.197 & -0.239
\end{array}\right] \text {, } \\
& b_{3}=10^{-1} \times[0.092] \text {. }
\end{aligned}
$$

\section{Conclusion}

To assess the feasibility of using Tabu Search Training (TST) algorithm to train Artificial Neural Networks (ANNs) for predicting the shear strength of reinforced concrete specimens, 248 experimental test results were collected from published results. After training the artificial neural network model with the lowest MSE on test dataset was selected, a sensitivity analysis was conducted on the model, and to assess its accuracy, other ANN models trained using particle swarm optimization and imperialist competitive algorithm were employed. The analysis of the results suggests the following:

(1) The trained ANN-TST 2L (9-5) model predicted the shear strength more accurately than other artificial neural networks. The mean squared error and model efficiency of this model on test data were 2217.08 and 0.9475 , respectively.

(2) A multiple regression model was provided as an easy-to-use model to predict the shear strength.

(3) An imperialist competitive algorithm-based and a particle swarm optimization-based artificial neural network were trained using the same data. The comparison of ANN-TST 2L (9-5), ANN-ICA 2L (95), ANN-PSO 2L (9-5), ACI code, and multiple regression models suggests that the TST-based ANN is the most accurate, followed by particle swarm optimization-based and imperialist competitive algorithm-based ANNs, and then the multiple regression model.

(4) The shear design formula of ACI-318-2019 was also investigated, and its accuracy was compared with that of ANN-TST 2L (9-5) model. The Tabu Search based ANN was shown to be superior in accuracy.

(5) Sensitivity analysis results suggest that the top three most influential parameters on shear strength of reinforced concrete T-beams are flexural reinforcement ratio, effective depth, and web width. The least influential parameters are shear span-to-depth ratio and concrete compressive strength.

(6) A predictive model based on the weights and biases of the top trained model, ANN-TST 2L (9-5), was provided to make the trained model available without the need for a computer source file.

\section{Data Availability}

The datasets are available at https://onlinelibrary.wiley.com/ doi/abs/10.1002/suco.201700095. 


\section{Conflicts of Interest}

The authors declare that they have no conflicts of interest.

\section{Acknowledgments}

This research did not receive any specific grant from funding agencies in the public, commercial, or not-for-profit sectors.

\section{References}

[1] H. Baghi and J. A. O. Barros, "Design-oriented approach to predict shear strength of reinforced concrete beams," Structural Concrete, vol. 19, no. 1, pp. 98-115, 2018.

[2] J. Amani and R. Moeini, "Prediction of shear strength of reinforced concrete beams using adaptive neuro-fuzzy inference system and artificial neural network," Scientia Iranica, vol. 19, no. 2, pp. 242-248, 2012.

[3] M. Y. Mansour, M. Dicleli, J. Y. Lee, and J. Zhang, "Predicting the shear strength of reinforced concrete beams using artificial neural networks," Engineering Structures, vol. 26, no. 6, pp. 781-799, 2004.

[4] O. B. Olalusi and P. O. Awoyera, "Shear capacity prediction of slender reinforced concrete structures with steel fibers using machine learning," Engineering Structures, vol. 227, Article ID 111470, 2021.

[5] A. Cladera and A. R. Marí, "Shear design procedure for reinforced normal and high-strength concrete beams using artificial neural networks. Part II: beams with stirrups," Engineering Structures, vol. 26, no. 7, pp. 927-936, 2004.

[6] J. A. Abdalla, A. Elsanosi, and A. Abdelwahab, "Modeling and simulation of shear resistance of R/C beams using artificial neural network," Journal of the Franklin Institute, vol. 344, no. 5, pp. 741-756, 2007.

[7] M. H. Arslan, "Predicting of torsional strength of RC beams by using different artificial neural network algorithms and building codes," Advances in Engineering Software, vol. 41, no. 7-8, pp. 946-955, 2010.

[8] A. W. C. Oreta, "Simulating size effect on shear strength of RC beams without stirrups using neural networks," Engineering Structures, vol. 26, no. 5, pp. 681-691, 2004.

[9] H. Naderpour and K. Nagai, "Shear strength estimation of reinforced concrete beam-column sub-assemblages using multiple soft computing techniques," The Structural Design of Tall and Special Buildings, vol. 29, no. 9, Article ID e1730, 2020.

[10] D.-C. Feng and B. Fu, "Shear strength of internal reinforced concrete beam-column joints: intelligent modeling approach and sensitivity analysis," Advances in Civil Engineering, vol. 2020, Article ID 8850417, 19 pages, 2020.

[11] J.-S. Jeon, A. Shafieezadeh, and R. DesRoches, "Statistical models for shear strength of RC beam-column joints using machine-learning techniques," Earthquake Engineering \& Structural Dynamics, vol. 43, no. 14, pp. 2075-2095, 2014.

[12] H. Salehi and R. Burgueño, "Emerging artificial intelligence methods in structural engineering," Engineering Structures, vol. 171, pp. 170-189, 2018.

[13] D. Prayogo, M.-Y. Cheng, Y.-W. Wu, and D.-H. Tran, "Combining machine learning models via adaptive ensemble weighting for prediction of shear capacity of reinforcedconcrete deep beams," Engineering with Computers, vol. 36, no. 3, pp. 1135-1153, 2020.

[14] G. Zhang, Z. H. Ali, M. S. Aldlemy et al., "Reinforced concrete deep beam shear strength capacity modelling using an integrative bio-inspired algorithm with an artificial intelligence model," Engineering with Computers, 2020.

[15] M. Shahnewaz, A. Rteil, and M. S. Alam, "Shear strength of reinforced concrete deep beams - a review with improved model by genetic algorithm and reliability analysis," Structure, vol. 23, pp. 494-508, 2020.

[16] M. Pal and S. Deswal, "Support vector regression based shear strength modelling of deep beams," Computers \& Structures, vol. 89, no. 13-14, pp. 1430-1439, 2011.

[17] C. Jui-Sheng, N. Ngoc-Tri, and P. Anh-Duc, "Shear strength prediction in reinforced concrete deep beams using natureinspired metaheuristic support vector regression," Journal of Computing in Civil Engineering, vol. 30, no. 1, Article ID 4015002, 2016.

[18] A. H. Gandomi, A. H. Alavi, D. M. Shadmehri, and M. G. Sahab, "An empirical model for shear capacity of RC deep beams using genetic-simulated annealing," Archives of Civil and Mechanical Engineering, vol. 13, no. 3, pp. 354-369, 2013.

[19] A. Sanad and M. P. Saka, "Prediction of ultimate shear strength of reinforced-concrete deep beams using neural networks," Journal of Structural Engineering, vol. 127, no. 7, pp. 818-828, 2001.

[20] N. Aalimahmoody, C. Bedon, N. Hasanzadeh-Inanlou, A. Hasanzade-Inallu, and M. Nikoo, "BAT algorithm-based ANN to predict the compressive strength of concrete-a comparative study," Infrastructure, vol. 6, no. 6, p. 80, 2021.

[21] S. Shekhar, M. B. Amin, and P. Khandelwal, Generalization Performance of Feed-Forward Neural Networks, E. B. T.-N. N. Gelenbe, Ed., North-Holland, Amsterdam, 1992.

[22] G. Ellis, "Feed-forward," in Control System Design GuideButterworth-Heinemann, Oxford, United Kingdom, 2004.

[23] A. Pourdaryaei, H. Mokhlis, H. A. Illias, S. H. A. Kaboli, S. Ahmad, and S. P. Ang, "Hybrid ANN and artificial cooperative search algorithm to forecast short-term electricity price in de-regulated electricity market," IEEE Access, vol. 7, pp. 125369-125386, 2019.

[24] M. Fan, Z. Zhang, and C. Wang, "Optimization method for load frequency feed forward control," in Mathematical Models and Algorithms for Power System Optimization, Elsevier Science, Amsterdam, The Netherlands, 2019.

[25] S. Haykin, Neural Networks and Learning Machines, Vol. 3, Pearson Education India, , London, United Kingdom, 2008.

[26] A. Géron, Hands-on Machine Learning with Scikit-Learn and TensorFlow: Concepts, Tools, and Techniques to Build Intelligent Systems, O’Reilly Media, Inc., Newton, Massachusetts, United States, 2017.

[27] M. Nikoo, Ł. Sadowski, F. Khademi, and M. Nikoo, "Determination of damage in reinforced concrete frames with shear walls using self-organizing feature map," Applied Computational Intelligence and Soft Computing, vol. 2017, Article ID 3508189, 10 pages, 2017.

[28] F. Glover, "Future paths for integer programming and links to artificial intelligence," Computers \& Operations Research, vol. 13, 1986.

[29] F. Glover, "Tabu search-Part I," ORSA Journal on Computing, vol. 1, no. 3, pp. 190-206, 1989.

[30] F. Glover, "Tabu search-Part II," ORSA Journal on Computing, vol. 2, no. 1, pp. 4-32, 1990.

[31] B. Dengiz, C. Alabas-Uslu, and O. Dengiz, "A tabu search algorithm for the training of neural networks," Journal of the Operational Research Society, vol. 60, no. 2, pp. 282-291, 2009. 
[32] O. Hajji, S. Brisset, and P. Brochet, "A new tabu search method for optimization with continuous parameters," IEEE Transactions on Magnetics, vol. 40, no. 2, pp. 1184-1187, 2004.

[33] X.-S. Yang, "Metaheuristic optimization: nature-inspired algorithms and applications," Studies in Computational Intelligence, vol. 427, pp. 405-420, 2013.

[34] S. J. E. Dias and J. A. O. Barros, "Shear strengthening of RC beams with NSM CFRP laminates: experimental research and analytical formulation," Composite Structures, vol. 99, pp. 477-490, 2013.

[35] P. Y. Kong and B. V. Rangan, "Shear strength of high-performance concrete beams," ACI Structural Journal, vol. 95, no. 6,1998

[36] R. J. Frosch, "Behavior of large-scale reinforced concrete beams with minimum shear reinforcement," ACI Structural Journal, vol. 97, no. 6, 2000.

[37] W. D. C. Young-Soo Yoon and D. Mitchell, "Minimum shear reinforcement in normal, medium, and high-strength concrete beams," ACI Structural Journal, vol. 93, no. 5, 1996.

[38] J. J. Roller and H. G. Russel, "Shear strength of high-strength concrete beams with web reinforcement," ACI Structural Journal, vol. 87, no. 2, 1990.

[39] K.-H. Reineck, E. Bentz, B. Fitik, D. A. Kuchma, and O. Bayrak, "ACI-DAfStb databases for shear tests on slender reinforced concrete beams with stirrups," ACI Structural Journal, vol. 111, no. 5, 5 pages, 2014.

[40] A. G. Mphonde, "Use of stirrup effectiveness in shear design of concrete beams," ACI Structural Journal, vol. 86, no. 5, 1989.

[41] U. E. Guney Ozcebe and T. Tankut, "Evaluation of minimum shear reinforcement requirements for higher strength concrete," ACI Structural Journal, vol. 96, no. 3, 1999.

[42] B. Bresler and A. C. Scordelis, "Shear strength of reinforced concrete beams," ACI Journal Proceedings, vol. 60, no. 1, 1963.

[43] I.-J. C. Jung-Yoon Lee and S.-W. Kim, "Shear behavior of reinforced concrete beams with high-strength stirrups," ACI Structural Journal, vol. 108, no. 5, 2011.

[44] W. J. Krefeld and C. W. Thurston, "Studies of the shear and diagonal tension strength of simply supported reinforced concrete beams," ACI Journal Proceedings, vol. 63, no. 4, 1966.

[45] L. Vandewalle, D. Nemegeer, G. Balázs, and B. Barr, "Rilem tc 162-TDF: 'test and design methods for steel fibre reinforced concrete' $\sigma$ - $\varepsilon$-design method," Mater. Struct. Constr.vol. 36, no. 262, pp. 560-567, 2003.

[46] A. Hasanzade-Inallu, P. Zarfam, and M. Nikoo, "Modified imperialist competitive algorithm-based neural network to determine shear strength of concrete beams reinforced with FRP," Journal of Central South University, vol. 26, no. 11, pp. 3156-3174, 2019.

[47] J. Li and A. D. Heap, A Review of Spatial Interpolation Methods for Environmental Scientists, Australian Gvernment, Canberra, 2008.

[48] A. Hasanzade-Inallu, P. Hassanzadeh Inallou, and B. Eskandarinezhad, "Prediction of compressive strength of concrete with manufactured sand using neural networks and bat algorithm," Soil Struct. Interact. J.vol. 4, pp. 52-63, 2019.

[49] G. J. Bowden, G. C. Dandy, and H. R. Maier, "Input determination for neural network models in water resources applications. Part 1-background and methodology," Journal of Hydrology, vol. 301, no. 1-4, pp. 75-92, 2005.

[50] Matlab, Mathworks, Mathematical Computing Software, Natick, Massachusetts, U.S.A, 2018.

[51] M. Nikoo, F. Torabian Moghadam, and Ł. Sadowski, "Prediction of concrete compressive strength by evolutionary artificial neural networks," Advances in Materials Science and Engineering, vol. 2015, Article ID 849126, 8 pages, 2015.

[52] D. K. Panesar, M. Aqel, D. Rhead, and H. Schell, "Effect of cement type and limestone particle size on the durability of steam cured self-consolidating concrete," Cement and Concrete Composites, vol. 80, pp. 175-189, 2017.

[53] M. Gevrey, I. Dimopoulos, and S. Lek, "Review and comparison of methods to study the contribution of variables in artificial neural network model," Ecological Modelling, vol. 160, 2003.

[54] I. Faridmehr, M. Nikoo, M. H. Baghban, and R. Pucinotti, "Hybrid krill herd-ANN model for prediction strength and stiffness of bolted connections," Buildings, vol. 11, no. 6, pp. 229-6, 2021.

[55] S. Lek, M. Delacoste, P. Baran, I. Dimopoulos, J. Lauga, and S. Aulagnier, "Application of neural networks to modelling nonlinear relationships in ecology," Ecological Modelling, vol. 90, no. 1, pp. 39-52, 1996. 